SUPPORTING INFORMATION FOR

\title{
Iron-Catalyzed Imination of Sulfoxides and Sulfides
}

\author{
Olga García Mancheño and Carsten Bolm* \\ Institute of Organic Chemistry, RWTH Aachen University, Landoltweg 1, D-52056 \\ Aachen, Germany \\ e-mail: Carsten.Bolm@oc.rwth-aachen.de
}

Table of Contents

- General

- General procedure for the imination reaction

- Analytical data for compound 1-16

$\mathrm{S} 2$

- Deprotection of $N$-(4-nitro-benzenesulfonyl) methyl phenyl sulfoximine

S5

- References

S6

- ${ }^{1} \mathrm{H}$ and ${ }^{13} \mathrm{C}$ spectra for all products

S7

General: Microanalyses were obtained with a Vario EL element analyzer. Mass spectra were acquired on a Finnigan SSQ7000 (CI $100 \mathrm{eV}$ ) spectrometer. IR spectra were taken on a Perkin-Elmer FT/IR 1760 and were recorded as $\mathrm{KBr}$ pellets. ${ }^{1} \mathrm{H}$ and ${ }^{13} \mathrm{C}$ NMR spectra were recorded in $\mathrm{CDCl}_{3}$ or DMSO-d $\mathrm{d}_{6}$ on a Varian Inova 400 or a Varian Mercury 300 spectrometer (400 and $100 \mathrm{MHz}$, and 300 and $75 \mathrm{MHz}$, respectively). Chemical shifts are given in ppm and spin-spin coupling constants, $J$, are given in Hz. Melting points were determined in open-end capillary tubes on a Büchi B-540 melting point apparatus and are uncorrected. HighPerformance Liquid Chromatography (HPLC) was conducted on a Gynkotek M480 instrument, using Daicel Chiralcel OD and OJ columns. Iodosylbenzene, $N$-( $p$-nosyl)imido phenyliodinane and [I,I-bis(trifluoroacetoxy)iodo]benzene were prepared from $\mathrm{PhI}(\mathrm{OAc})_{2}$, and 1,4-bis(diacetoxyiodo)benzene was synthesized from 1,4-diiodobenzene according to literature procedures. ${ }^{1}$ 
General procedure for the imination reaction: A mixture of sulfoxide or sulfide $(0.285$ mmol), sulfonyl amide $(0.423 \mathrm{mmol})$, iodosylbenzene $(100.3 \mathrm{mg}, 0.456 \mathrm{mmol})$ and $\mathrm{Fe}(\mathrm{acac})_{3}$ (5.0 mg, $0.014 \mathrm{mmol})$ in $\mathrm{CH}_{3} \mathrm{CN}(3 \mathrm{~mL})$ was stirred at room temperature. Once the starting material was consumed (monitored by TLC), the reaction mixture was concentrated to half its volume under reduced pressure. The residue was purified by flash column chromatography (the eluent is indicated in each case) to afford the corresponding sulfoximines or sulfilimines.

$\boldsymbol{N}$-(4-Nitro-benzenesulfonyl) methyl phenyl sulfoximine (2): ${ }^{2}$ Following the general procedure, the reaction of methyl phenyl sulfoxide with $\mathrm{NsNH}_{2}$ gave 2 as a pale yellow solid (93.1 mg, 96\%). Chromatography: gradient of ethyl acetate/pentane 1:2 to 1:1. Mp. 148-150 ${ }^{\circ} \mathrm{C}$ (Lit. ${ }^{2 \mathrm{c}} 148-151{ }^{\circ} \mathrm{C}$ ); ${ }^{1} \mathrm{H}$ NMR (400 MHz, $\mathrm{CDCl}_{3}$ ): $\delta 8.32$ (br d, $\left.J=9.2 \mathrm{~Hz}, 2 \mathrm{H}\right), 8.16$ (br d, $J=9.2 \mathrm{~Hz}, 2 \mathrm{H}), 8.03$ (br d, $J=7.9 \mathrm{~Hz}, 2 \mathrm{H}), 7.80-7.72(\mathrm{~m}, 1 \mathrm{H}), 7.68-7.62(\mathrm{~m}, 2 \mathrm{H}), 3.47$ (s, $3 \mathrm{H}) ;{ }^{13} \mathrm{C} \mathrm{NMR}\left(100 \mathrm{MHz}, \mathrm{CDCl}_{3}\right): \delta 149.7(\mathrm{C}), 149.0(\mathrm{C}), 137.8(\mathrm{C}), 134.9(\mathrm{CH}), 130.0(2 \mathrm{x}$ $\mathrm{CH}), 128.1(2 \times \mathrm{CH}), 127.4(2 \times \mathrm{CH}), 124.0(2 \times \mathrm{CH}), 46.9\left(\mathrm{CH}_{3}\right)$.

$N$-(p-Toluenesulfonyl) methyl phenyl sulfoximine (3): ${ }^{2 \mathrm{a}, 3}$ Following the general procedure, the reaction of methyl phenyl sulfoxide with $\mathrm{TsNH}_{2}$ gave 3 as a white solid $(71.6 \mathrm{mg}, 81 \%)$. Chromatography: gradient of ethyl acetate/pentane $1: 2$ to $1: 1$. Mp. $108-109^{\circ} \mathrm{C}$ (Lit. ${ }^{3 b} 107-109$ $\left.{ }^{\circ} \mathrm{C}\right) ;{ }^{1} \mathrm{H} \mathrm{NMR}\left(300 \mathrm{MHz}, \mathrm{CDCl}_{3}\right): \delta 8.03$ (br d, $\left.J=9.1 \mathrm{~Hz}, 2 \mathrm{H}\right), 7.87$ (br d, $\left.J=9.1 \mathrm{~Hz}, 2 \mathrm{H}\right)$, 7.76-7.68 (m, 1H), 7.66-7.58 (m, 2H), 7.32-7.24 (m, 2H), 3.44 (s, 3H), 2.41 (s, 3H); ${ }^{13} \mathrm{C}$ NMR $\left(75 \mathrm{MHz}, \mathrm{CDCl}_{3}\right): \delta 142.9(\mathrm{C}), 140.7(\mathrm{C}), 138.4(\mathrm{C}), 134.4(\mathrm{CH}), 129.7(2 \mathrm{x} \mathrm{CH}), 129.3(2 \mathrm{x}$ $\mathrm{CH}), 127.5(2 \times \mathrm{CH}), 126.7(2 \times \mathrm{CH}), 46.7\left(\mathrm{CH}_{3}\right), 21.5\left(\mathrm{CH}_{3}\right)$.

$N$-(Trimethylsilylethylsulfonyl) methyl phenyl sulfoximine (4): ${ }^{2 a}$ Following the general procedure, the reaction of methyl phenyl sulfoxide with $\mathrm{SES}-\mathrm{NH}_{2}{ }^{4}$ gave 4 as a white solid (61.5 mg, 66\%). Chromatography: ethyl acetate/pentane 1:2. Mp. 107-109 ${ }^{\circ} \mathrm{C}$ (Lit. ${ }^{2 \mathrm{a}} 106-108$ $\left.{ }^{\circ} \mathrm{C}\right) ;{ }^{1} \mathrm{H}$ NMR (400 MHz, $\mathrm{CDCl}_{3}$ ): $\delta 8.01$ (br d, $\left.J=7.9 \mathrm{~Hz}, 2 \mathrm{H}\right), 7.69$ (br t, $J=7.4 \mathrm{~Hz}, 1 \mathrm{H}$ ), 7.59 (br t, $J=7.9 \mathrm{~Hz}, 2 \mathrm{H}), 3.40$ (s, 3H), 3.15-3.05 (m, 2H), 1.20-1.00 (m, 2H), 0.00 (s, 9H); ${ }^{13} \mathrm{C} \mathrm{NMR}\left(100 \mathrm{MHz}, \mathrm{CDCl}_{3}\right): \delta 138.5(\mathrm{C}), 134.5(\mathrm{CH}), 129.8(2 \times \mathrm{CH}), 127.4(2 \times \mathrm{CH}), 53.6$ $\left(\mathrm{CH}_{2}\right), 46.6\left(\mathrm{CH}_{3}\right), 10.4\left(\mathrm{CH}_{2}\right), 2.0\left(\mathrm{CH}_{3}\right)$.

$N$-(4-Methyl-2-pyridinylsulfonyl) methyl phenyl sulfoximine (5): ${ }^{2 a}$ Following the general procedure, the reaction of methyl phenyl sulfoxide with 4-methyl-2-pyridinyl sulfonyl amide gave 5 as a colourless oil (79.0 mg, 87\%). Chromatography: ethyl acetate/pentane 1:1. ${ }^{1} \mathrm{H}$ NMR (300 MHz, $\mathrm{CDCl}_{3}$ ): $\delta 8.43$ (br s, 1H), 7.97-7.90 (m, 2H), 7.85 (d, J=7.9 Hz, 1H), 7.65- 
$7.55(\mathrm{~m}, 2 \mathrm{H}), 7.51(\mathrm{t}, J=7.9 \mathrm{~Hz}, 2 \mathrm{H}), 3.50(\mathrm{~s}, 3 \mathrm{H}), 2.33(\mathrm{~s}, 3 \mathrm{H}) ;{ }^{13} \mathrm{C} \operatorname{NMR}(75 \mathrm{MHz}$, $\left.\mathrm{CDCl}_{3}\right): \delta 157.0(\mathrm{C}), 150.1(\mathrm{CH}), 138.3(\mathrm{C}), 138.0(\mathrm{CH}), 137.0(\mathrm{C}), 134.4(\mathrm{CH}), 129.7(2 \mathrm{x}$ $\mathrm{CH}), 127.5(2 \times \mathrm{CH}), 121.3(\mathrm{CH}), 46.3\left(\mathrm{CH}_{3}\right), 18.5\left(\mathrm{CH}_{3}\right)$.

$N$-(2-Benzothiazolesulfonyl) methyl phenyl sulfoximine (6): Following the general procedure, the reaction of methyl phenyl sulfoxide with 2-benzothiazolesulfonyl amide gave $^{5}$ 6 as a white solid (88.2 mg, 88\%). Chromatography: gradient of ethyl acetate/pentane 1:2 to 1:1. Mp. 131-133 ${ }^{\circ} \mathrm{C} ;{ }^{1} \mathrm{H}$ NMR (400 MHz, $\left.\mathrm{CDCl}_{3}\right): \delta 8.13-8.07(\mathrm{~m}, 1 \mathrm{H}), 7.99-7.94(\mathrm{~m}, 2 \mathrm{H})$, 7.89-7.83 (m, 1H), 7.66-7.60 (m, 1H), 7.56-7.42 (m, 4H), 3.52 (s, 3H); ${ }^{13} \mathrm{C}$ NMR (100 MHz, $\left.\mathrm{CDCl}_{3}\right): \delta 152.1(\mathrm{C}), 137.6(\mathrm{C}), 136.6(\mathrm{C}), 134.8(\mathrm{CH}), 129.8(2 \times \mathrm{CH}), 127.5(2 \times \mathrm{CH}), 127.4$ $(\mathrm{CH}), 127.1(\mathrm{CH}), 125.3(\mathrm{CH}), 122.1(\mathrm{CH}), 114.5(\mathrm{C}), 46.6\left(\mathrm{CH}_{3}\right)$; IR $(\mathrm{KBr})$ : v 3016, 2926, 1470, 1322, 1233, 1152, 1094, $1058 \mathrm{~cm}^{-1}$; MS (CI), m/z (relative intensity): 353 [M $\left.{ }^{+}, 100\right]$; Calcd. for $\mathrm{C}_{14} \mathrm{H}_{12} \mathrm{~N}_{2} \mathrm{O}_{3} \mathrm{~S}_{3}$ : C, 47.71; H, 3.43; N, 7.95; found C, 47.89; H, 3.82; N, 7.81 .

$N$-(4-Nitro-benzenesulfonyl)-tert-butyl methyl sulfoximine (7): ${ }^{2 \mathrm{c}}$ Following the general procedure, the reaction of tert-butyl methyl sulfoxide with $\mathrm{NsNH}_{2}$ gave 7 as a white solid (72.9 mg, 80\%). Chromatography: dichloromethane. Mp. 146-147 ${ }^{\circ} \mathrm{C}$ (decomp.) [Lit. ${ }^{2 \mathrm{c}} 156$ ${ }^{\circ} \mathrm{C}$ (decomp.)]; ${ }^{1} \mathrm{H}$ NMR (400 MHz, $\mathrm{CDCl}_{3}$ ): $\delta 8.25$ (br d, $J=9.1 \mathrm{~Hz}, 2 \mathrm{H}$ ), 8.08 (br d, $J=9.1$ $\mathrm{Hz}, 2 \mathrm{H}), 3.33$ (s, 3H), $1.40(\mathrm{~s}, 9 \mathrm{H}) ;{ }^{13} \mathrm{C} \mathrm{NMR}\left(100 \mathrm{MHz}, \mathrm{CDCl}_{3}\right): \delta 149.6(\mathrm{C}), 149.4(\mathrm{C})$, $127.8(2 \times \mathrm{CH}), 123.9(2 \times \mathrm{CH}), 62.5(\mathrm{C}), 35.5\left(\mathrm{CH}_{3}\right), 22.8\left(3 \times \mathrm{CH}_{3}\right)$.

$\boldsymbol{N}$-(4-Nitro-benzenesulfonyl) diphenyl sulfoximine (8): ${ }^{2 \mathrm{a}, \mathrm{c}}$ Following the general procedure, the reaction of diphenyl sulfoxide with $\mathrm{NsNH}_{2}$ gave 8 as a white solid $(91.8 \mathrm{mg}, 80 \%)$. Chromatography: dichloromethane. Mp. 154-155 ${ }^{\circ} \mathrm{C}$ (Lit. $\left.{ }^{2 \mathrm{c}} 154-156{ }^{\circ} \mathrm{C}\right) ;{ }^{1} \mathrm{H}$ NMR $(300 \mathrm{MHz}$, $\mathrm{CDCl}_{3}$ ): $\delta 8.22$ (br d, $\left.J=8.9 \mathrm{~Hz}, 2 \mathrm{H}\right), 8.09$ (br d, $\left.J=8.9 \mathrm{~Hz}, 2 \mathrm{H}\right), 7.97-7.90$ (m, 4H), 7.61$7.43(\mathrm{~m}, 6 \mathrm{H}) ;{ }^{13} \mathrm{C} \mathrm{NMR}\left(75 \mathrm{MHz}, \mathrm{CDCl}_{3}\right): \delta 149.7$ (C), $149.2(\mathrm{C}), 139.4(2 \mathrm{x} \mathrm{C}), 134.2(2 \mathrm{x}$ $\mathrm{CH}), 129.8$ (4 x CH), $128.1(2 \times \mathrm{CH}), 127.6(4 \times \mathrm{CH}), 124.0(2 \times \mathrm{CH})$.

$N$-(4-Nitro-benzenesulfonyl) phenyl vinyl sulfoximine (9): ${ }^{2 a}$ Following the general procedure, the reaction of phenyl vinyl sulfoxide with $\mathrm{NsNH}_{2}$ gave $\mathbf{9}$ as a pale yellow solid (72.1 mg, 72\%). Chromatography: dichloromethane. Mp. 149-150 ${ }^{\circ} \mathrm{C}$ (Lit. ${ }^{2 \mathrm{a}} 150-151{ }^{\circ} \mathrm{C}$ ); ${ }^{1} \mathrm{H}$ $\operatorname{NMR}\left(400 \mathrm{MHz}, \mathrm{CDCl}_{3}\right.$ ): $\delta 8.31$ (br d, $\left.J=9.1 \mathrm{~Hz}, 2 \mathrm{H}\right), 8.16$ (br d, $\left.J=9.1 \mathrm{~Hz}, 2 \mathrm{H}\right), 8.00-7.94$ $(\mathrm{m}, 2 \mathrm{H}), 7.72(\mathrm{tt}, J=7.6,1.1 \mathrm{~Hz}, 1 \mathrm{H}), 7.65-7.56(\mathrm{~m}, 2 \mathrm{H}), 6.84(\mathrm{dd}, J=16.2,9.6 \mathrm{~Hz}, 1 \mathrm{H})$, $6.50(\mathrm{dd}, J=16.2,1.6 \mathrm{~Hz}, 1 \mathrm{H}), 6.24(\mathrm{dd}, J=9.6,1.6 \mathrm{~Hz}, 1 \mathrm{H}) ;{ }^{13} \mathrm{C} \mathrm{NMR}\left(100 \mathrm{MHz}, \mathrm{CDCl}_{3}\right)$ : 
$\delta 149.7(\mathrm{C}), 149.0(\mathrm{C}), 137.2(\mathrm{CH}), 136.8(\mathrm{C}), 134.7(\mathrm{CH}), 129.9(2 \times \mathrm{CH}), 129.8\left(\mathrm{CH}_{2}\right)$, $128.0(2 \times \mathrm{CH}), 127.9(2 \times \mathrm{CH}), 124.0(2 \times \mathrm{CH})$.

$N$-(4-Nitro-benzenesulfonyl) tetramethylene sulfoximine (10): ${ }^{2 a, b}$ Following the general procedure, the reaction of tetramethylene sulfoxide with $\mathrm{NsNH}_{2}$ gave $\mathbf{1 0}$ as a pale yellow solid (74.6 mg, 86\%). Chromatography: ethyl acetate/pentane 1:1. Mp. 218-219 ${ }^{\circ} \mathrm{C}$ (Lit. ${ }^{2 \mathrm{a}}$ 222-225 ${ }^{\circ} \mathrm{C}$ ); ${ }^{1} \mathrm{H}$ NMR (400 MHz, DMSO-d 6 ): $\delta 8.35$ (br d, $\left.J=9.1 \mathrm{~Hz}, 2 \mathrm{H}\right), 8.06$ (br d, $J=$ 9.1 Hz, 2H), 3.74-3.64 (m, 2H), 3.52-3.42 (m, 2H), 2.25-2.05 (m, 4H); ${ }^{13} \mathrm{C}$ NMR (100 MHz, DMSO-d $\left.)_{6}\right): \delta 150.0(\mathrm{C}), 149.3(\mathrm{C}), 128.1(2 \times \mathrm{CH}), 124.8(2 \times \mathrm{CH}), 54.3\left(2 \mathrm{x} \mathrm{CH}_{2}\right), 23.2(2 \mathrm{x}$ $\mathrm{CH}_{2}$ ).

$N$-(4-Nitro-benzenesulfonyl) methyl phenyl sulfilimine (12): ${ }^{2}$ Following the general procedure, the reaction of methyl phenyl sulfide with $\mathrm{NsNH}_{2}$ gave 12 as a white solid (84.5 mg, 91\%). Chromatography: gradient of ethyl acetate/pentane 1:1 to ethyl acetate. Mp. 164$165{ }^{\circ} \mathrm{C}$ (Lit. $\left.{ }^{2 \mathrm{c}} 164.5-165.5{ }^{\circ} \mathrm{C}\right) ;{ }^{1} \mathrm{H}$ NMR $\left(300 \mathrm{MHz}, \mathrm{CDCl}_{3}\right): \delta 8.14$ (br d, $\left.J=9.2 \mathrm{~Hz}, 2 \mathrm{H}\right)$, 7.93 (br d, $J=9.2 \mathrm{~Hz}, 2 \mathrm{H}), 7.65-7.60(\mathrm{~m}, 2 \mathrm{H}), 7.54-7.40(\mathrm{~m}, 3 \mathrm{H}), 2.86(\mathrm{~s}, 3 \mathrm{H}) ;{ }^{13} \mathrm{C} \mathrm{NMR}(75$ $\left.\mathrm{MHz}, \mathrm{CDCl}_{3}\right): \delta 149.8(\mathrm{C}), 149.2(\mathrm{C}), 135.1(\mathrm{C}), 133.1(\mathrm{CH}), 130.3(2 \times \mathrm{CH}), 127.5$ (2 x CH), $125.9(2 \times \mathrm{CH}), 124.0(2 \times \mathrm{CH}), 39.2\left(\mathrm{CH}_{3}\right)$.

$N$-(4-Nitro-benzenesulfonyl) tert-butyl methyl sulfilimine (13): ${ }^{2 \mathrm{a}, \mathrm{c}}$ Following the general procedure, the reaction of tert-butyl methyl sulfide with $\mathrm{NsNH}_{2}$ gave $\mathbf{1 3}$ as a white solid (76.4 mg, 88\%). Chromatography: ethyl acetate. Mp. 134-135 ${ }^{\circ} \mathrm{C}\left[\right.$ Lit. $^{2 \mathrm{c}} 136{ }^{\circ} \mathrm{C}$ (decomp.)]; ${ }^{1} \mathrm{H}$ NMR (300 MHz, $\mathrm{CDCl}_{3}$ ): $\delta 8.29$ (br d, $\left.J=9.2 \mathrm{~Hz}, 2 \mathrm{H}\right), 8.07$ (br d, $\left.J=9.2 \mathrm{~Hz}, 2 \mathrm{H}\right), 2.49$ (s, 3H), 1.29 (s, 9H); ${ }^{13} \mathrm{C}$ NMR (75 MHz, $\left.\mathrm{CDCl}_{3}\right): \delta 150.1(\mathrm{C}), 149.1$ (C), 127.5 (2 x CH), 124.0 $(2 \times \mathrm{CH}), 55.7(\mathrm{C}), 28.0\left(\mathrm{CH}_{3}\right), 23.4\left(3 \mathrm{x} \mathrm{CH}_{3}\right)$.

$N$-(4-Nitro-benzensulfonyl) diphenyl sulfilimine (14): ${ }^{2 \mathrm{c}}$ Following the general procedure, the reaction of diphenyl sulfide with $\mathrm{NsNH}_{2}$ gave 14 as a white solid (100.0 mg, 91\%). Chromatography: gradient of ethyl acetate/pentane $1: 2$ to $1: 1$. Mp. $157-158{ }^{\circ} \mathrm{C}$ (Lit. $^{2 \mathrm{c}} 160.5-$ $161.5{ }^{\circ} \mathrm{C}$ ); ${ }^{1} \mathrm{H}$ NMR $\left(400 \mathrm{MHz}, \mathrm{CDCl}_{3}\right.$ ): $\delta 8.11$ (br d, $\left.J=8.8 \mathrm{~Hz}, 2 \mathrm{H}\right), 7.95$ (br d, $J=8.8 \mathrm{~Hz}$, 2H), 7.59-7.54 (m, 4H), 7.50-7.38 (m, 6H); ${ }^{13} \mathrm{C}$ NMR (100 MHz, $\left.\mathrm{CDCl}_{3}\right): \delta 149.9(\mathrm{C}), 149.1$ (C), $135.8(2 \times \mathrm{C}), 132.7(2 \times \mathrm{CH}), 130.1(2 \times \mathrm{CH}), 127.4(4 \times \mathrm{CH}), 127.2(4 \times \mathrm{CH}), 123.9(2$ $\mathrm{x} \mathrm{CH})$.

$N$-(4-Nitro-benzensulfonyl) phenyl allyl sulfilimine (15): Following the general procedure, the reaction of phenyl allyl sulfide with $\mathrm{NsNH}_{2}$ gave 15 as a white solid $(92.1 \mathrm{mg}, 92 \%)$. 
Chromatography: gradient of dichloromethane/pentane 1:2 to dichloromethane. Mp. 107-109 ${ }^{\circ} \mathrm{C} ;{ }^{1} \mathrm{H}$ NMR (400 MHz, $\mathrm{CDCl}_{3}$ ): $\delta 8.34$ (br d, $\left.J=9.1 \mathrm{~Hz}, 2 \mathrm{H}\right), 8.11$ (br d, $\left.J=9.1 \mathrm{~Hz}, 2 \mathrm{H}\right)$, 7.45-7.39 (m, 2H), 7.37-7.22 (m, 3H), 5.79-5.65 (m, 1H), 5.25-5.17 (m, 2H), 4.25-4.20 (m, $2 \mathrm{H}) ;{ }^{13} \mathrm{C} \mathrm{NMR}\left(100 \mathrm{MHz}, \mathrm{CDCl}_{3}\right): \delta 150.2(\mathrm{C}), 144.8(\mathrm{C}), 135.8(\mathrm{C}), 131.6(\mathrm{CH}), 129.2(2 \mathrm{x}$ $\mathrm{CH}), 129.1(2 \times \mathrm{CH}), 128.3(\mathrm{CH}), 127.3(2 \times \mathrm{CH}), 124.2(2 \times \mathrm{CH}), 120.4\left(\mathrm{CH}_{2}\right), 57.5\left(\mathrm{CH}_{2}\right)$; IR (KBr): $v$ 3061, 1582, 1526, 1354, 1308, 1166, 845, 738, $687 \mathrm{~cm}^{-1}$; MS (CI), m/z (relative intensity): $351\left[\mathrm{M}^{+}+\mathrm{H}, 100\right], 164\left[\mathrm{M}^{+}-\mathrm{Ns}, 42\right]$; Calcd. for $\mathrm{C}_{15} \mathrm{H}_{14} \mathrm{~N}_{2} \mathrm{O}_{4} \mathrm{~S}_{2}: \mathrm{C}, 51.41 ; \mathrm{H}, 4.03$; $\mathrm{N}, 7.99$; found $\mathrm{C}, 51.33 ; \mathrm{H}, 4.20 ; \mathrm{N}, 8.02$.

$\mathrm{N}$-(4-Nitro-benzensulfonyl) 2,4,6-(trimethylphenyl) methyl sulfilimine (16): Following the general procedure, the reaction of 2,4,6-(trimethylphenyl) methyl sulfide with $\mathrm{NsNH}_{2}$ gave 16 as a white solid (77.3 mg, 74\%). Chromatography: ethyl acetate/pentane 1:1. Mp. $180-182{ }^{\circ} \mathrm{C}$; ${ }^{1} \mathrm{H}$ NMR (400 MHz, $\left.\mathrm{CDCl}_{3}\right): \delta 8.11($ br d, $J=9.1 \mathrm{~Hz}, 2 \mathrm{H}), 7.88($ br d, $J=9.1 \mathrm{~Hz}, 2 \mathrm{H}), 6.84$ (s, 2H), $3.06(\mathrm{~s}, 3 \mathrm{H}), 2.57$ (s, 6H), $2.26(\mathrm{~s}, 3 \mathrm{H}) ;{ }^{13} \mathrm{C} \mathrm{NMR}\left(100 \mathrm{MHz}, \mathrm{CDCl}_{3}\right): \delta 149.9(\mathrm{C})$, $148.8(\mathrm{C}), 144.1(\mathrm{C}), 140.2$ ( $2 \times \mathrm{C}), 131.4(2 \times \mathrm{CH}), 127.2(2 \times \mathrm{CH}), 123.6(2 \times \mathrm{CH}), 34.3$ $\left(\mathrm{CH}_{3}\right), 21.2\left(\mathrm{CH}_{3}\right), 20.2\left(2 \times \mathrm{CH}_{3}\right)$; IR $(\mathrm{KBr}): v 3057,2927,1600,1525,1456,1350,1288$, 1137, 945, 764, $611 \mathrm{~cm}^{-1}$; MS (CI), m/z (relative intensity): $367\left[\mathrm{M}^{+}+\mathrm{H}, 100\right], 167\left[\mathrm{M}^{+}+\mathrm{H}-\right.$ NNs, 28]; Calcd. for $\mathrm{C}_{16} \mathrm{H}_{18} \mathrm{~N}_{2} \mathrm{O}_{4} \mathrm{~S}_{2}: \mathrm{C}, 52.44 ; \mathrm{H}, 4.95 ; \mathrm{N}, 7.64$; found $\mathrm{C}, 52.46 ; \mathrm{H}, 5.06 ; \mathrm{N}$, 7.53 .

$\mathrm{N}$-(tert-Butylsulfonyl) methyl phenyl sulfilimine (17): Following the general procedure, the reaction of phenyl methyl sulfide with $\mathrm{BusNH}_{2}{ }^{6}$ gave 17 as a white solid (32.9 mg, 45\%). Chromatography: ethyl acetate. Mp. $138-139{ }^{\circ} \mathrm{C} ;{ }^{1} \mathrm{H}$ NMR (400 MHz, $\mathrm{CDCl}_{3}$ ): $\delta 7.87-7.79$ (m, 2H), 7.61-7.55 (m, 3H), 2.91 (s, 3H), 1.34 (s, 9H); $\left.{ }^{13} \mathrm{C} \mathrm{NMR} \mathrm{(100} \mathrm{MHz,} \mathrm{CDCl}_{3}\right): \delta 137.5$ (C), $132.4(\mathrm{CH}), 130.1(2 \times \mathrm{CH}), 125.7(2 \times \mathrm{CH}), 59.4(\mathrm{C}), 41.0\left(\mathrm{CH}_{3}\right), 24.9\left(3 \times \mathrm{CH}_{3}\right)$; IR (KBr): $v$ 3064, 2964, 1474, 1445, 1415, 1262, 1106, 1009, 969, 757, $666 \mathrm{~cm}^{-1}$; MS (CI), m/z (relative intensity): $260\left[\mathrm{M}^{+}+\mathrm{H}, 100\right]$; Calcd. for $\mathrm{C}_{11} \mathrm{H}_{17} \mathrm{NO}_{2} \mathrm{~S}_{2}: \mathrm{C}, 50.93 ; \mathrm{H}, 6.61 ; \mathrm{N}, 5.40$; found C, 50.90; H, 6.49; N, 5.31 .

Deprotection of $\mathrm{N}$-(4-nitro-benzenesulfonyl) methyl phenyl sulfoximine to give $\mathrm{NH}$ methyl phenyl sulfoximine (11): ${ }^{2,7}$ To a solution of compound 2 (300.4 mg, $\left.0.88 \mathrm{mmol}\right)$ in acetonitrile $(13 \mathrm{~mL})$ was added $\mathrm{Cs}_{2} \mathrm{CO}_{3}(521.0 \mathrm{mg}, 1.6 \mathrm{mmol})$ and thiophenol $(144 \mu \mathrm{L}, 1.4$ $\mathrm{mmol}$ ) at room temperature and the reaction mixture was stirred overnight. Water was added to the reaction mixture and the product was extracted with dichloromethane. The combined 
organic layers were dried over $\mathrm{MgSO}_{4}$ and concentrated. Purification by flash column chromatography (gradient of ethyl acetate to ethyl acetate $/ 20 \% \mathrm{EtOH}$ ) gave $\mathbf{1 1}$ as colorless oil (113.2 mg, 83\%). HPLC: Daicel Chiralcel OJ [heptane/i-PrOH 85:15; $0.5 \mathrm{~mL} / \mathrm{min} ; 254 \mathrm{~nm}$; $\left.(R)-2 t_{R}=36.9 \mathrm{~min} ;(S)-2 t_{R}=48.9 \mathrm{~min}\right] ;{ }^{1} \mathrm{H}$ NMR $\left(300 \mathrm{MHz}, \mathrm{CDCl}_{3}\right): \delta 8.05-8.00(\mathrm{~m}, 2 \mathrm{H})$, 7.69-7.52 (m, 3H), 3.11 (s, 3H), 2.47 (br s, $1 \mathrm{H}) ;{ }^{13} \mathrm{C} \mathrm{NMR}\left(75 \mathrm{MHz}, \mathrm{CDCl}_{3}\right): \delta 143.5(\mathrm{C})$, $133.1(\mathrm{CH}), 129.3(2 \times \mathrm{CH}), 127.7(2 \times \mathrm{CH}), 46.2\left(\mathrm{CH}_{3}\right)$.

\section{References}

1. Iodosylbenzene: (a) Dauban, P.; Sanière, L.; Tarrade, A.; Dodd, R. H. J. Am. Chem. Soc. 2001, 123, 7707. (b) Saltzman, H.; Sharefkin, J. G. Org. Synth. 1973, 5, 658. N-(pNosyl)imido phenyliodinane: (c) Taylor, S.; Gullick, J.; McMorn, P.; Bethell, D.; Page, P. C. B.; Hancock, F. E.; King, F.; Hutchings G. J. Topics in Catalysis 2003, 24, 43. [I,IBis(trifluoroacetoxy)iodo]benzene: (d) Loudon, G. M.; Radhakrishna, A. S.; Almond, M. R.; Blodgett, J. K.; Boutin R. H. J. Org. Chem. 1984, 49, 4212. 1,4-Bis(diacetoxyiodo)benzene: (e) Hossain, Md. D.; Kitamura T. J. Org. Chem. 2005, 70, 6984.

2. (a) Cho, G. Y.; Bolm, C. Org. Lett. 2005, 7, 4983. (b) Cho, G. Y.; Bolm, C. Tetrahedron Lett. 2005, 46, 8007. (c) Okamura, H.; Bolm, C. Org. Lett. 2004, 6, 1305.

3. (a) Akutagawa, K.; Furukawa, N. J. Org. Chem. 1984, 49, 2282. (b) Johnson, C. R.; Kirchhoff, R. A.; Reischer, R. J.; Katekarlb, G. F. J. Am. Chem. Soc. 1973, 95, 4287.

4. Declerck, V.; Ribiére, P.; Martinez, J.; Lamaty, F. J. Org. Chem. 2004, 69, 8372.

5. (a) Schoenwald, R. D.; Eller, M. G.; Dixson, J. A.; Barfknecht, C. F. J. Med. Chem. 1984, 27, 810. (b) Roblin, R. O.; Clapp, J. W. J. Am. Chem. Soc. 1950, 72, 4890.

6. (a) Schleusner, M.; Koep, S.; Günter, M.; Tiwari, S. K.; Gais, H.-J. Synthesis 2004, 967. (b) Gontcharov, A. V.; Liu, H.; Sharpless, K. B. Org. Lett. 1999, 1, 783

7. Cren, S.; Kinahan, T. C.; Skinner, C. L.; Tye, H. Tetrahedron Lett. 2002, 43, 2749. 

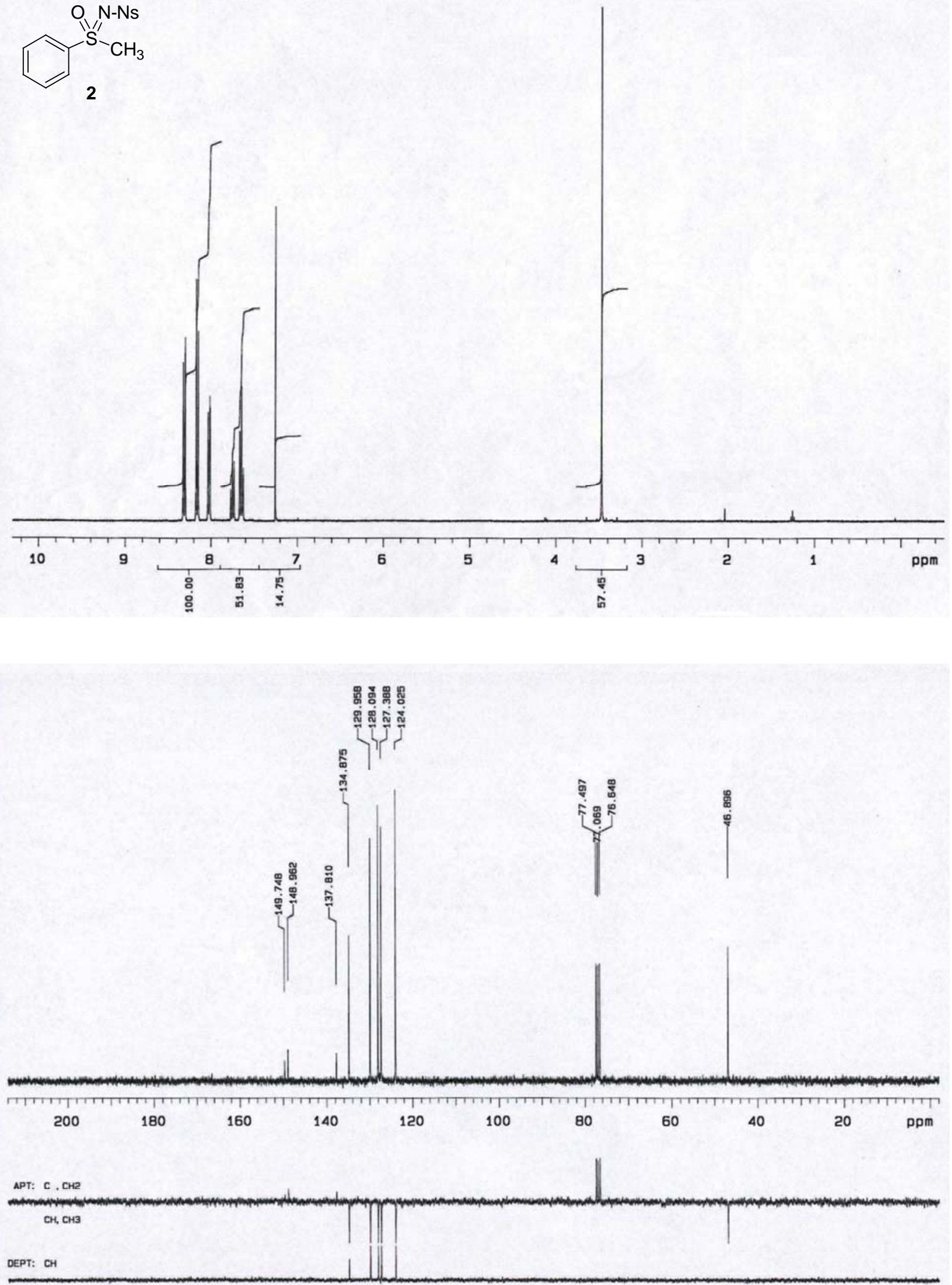


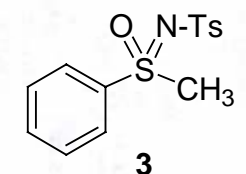

3
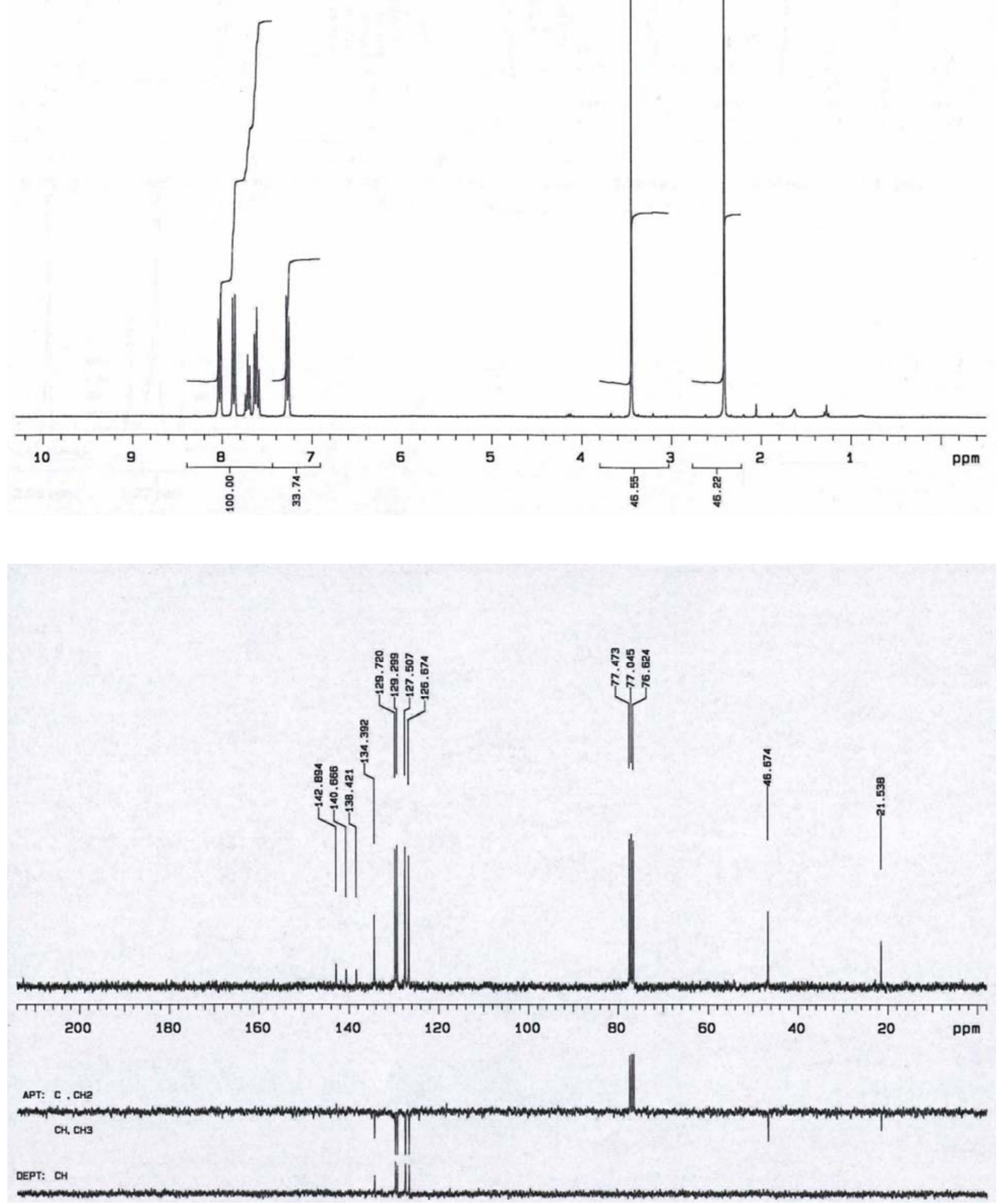

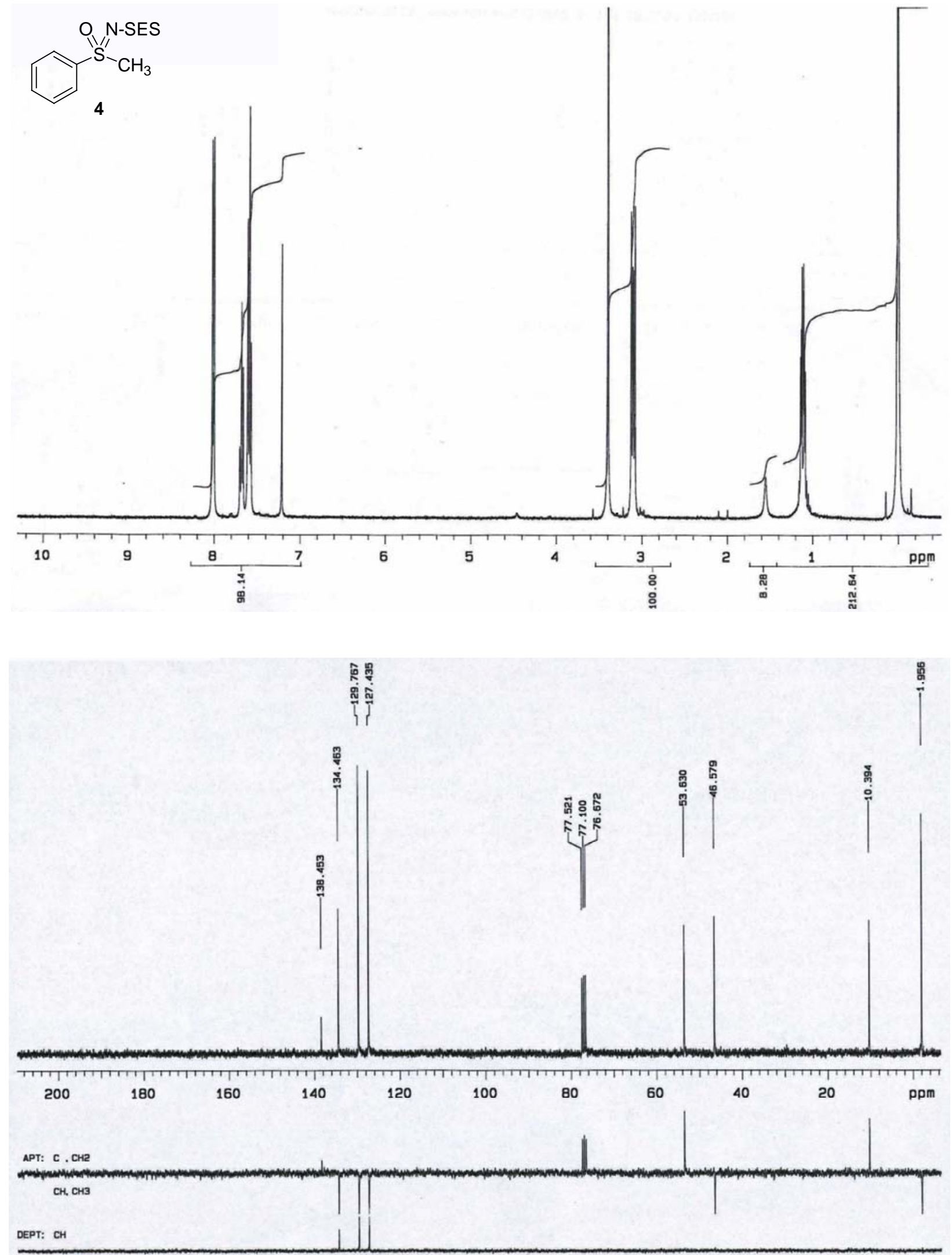

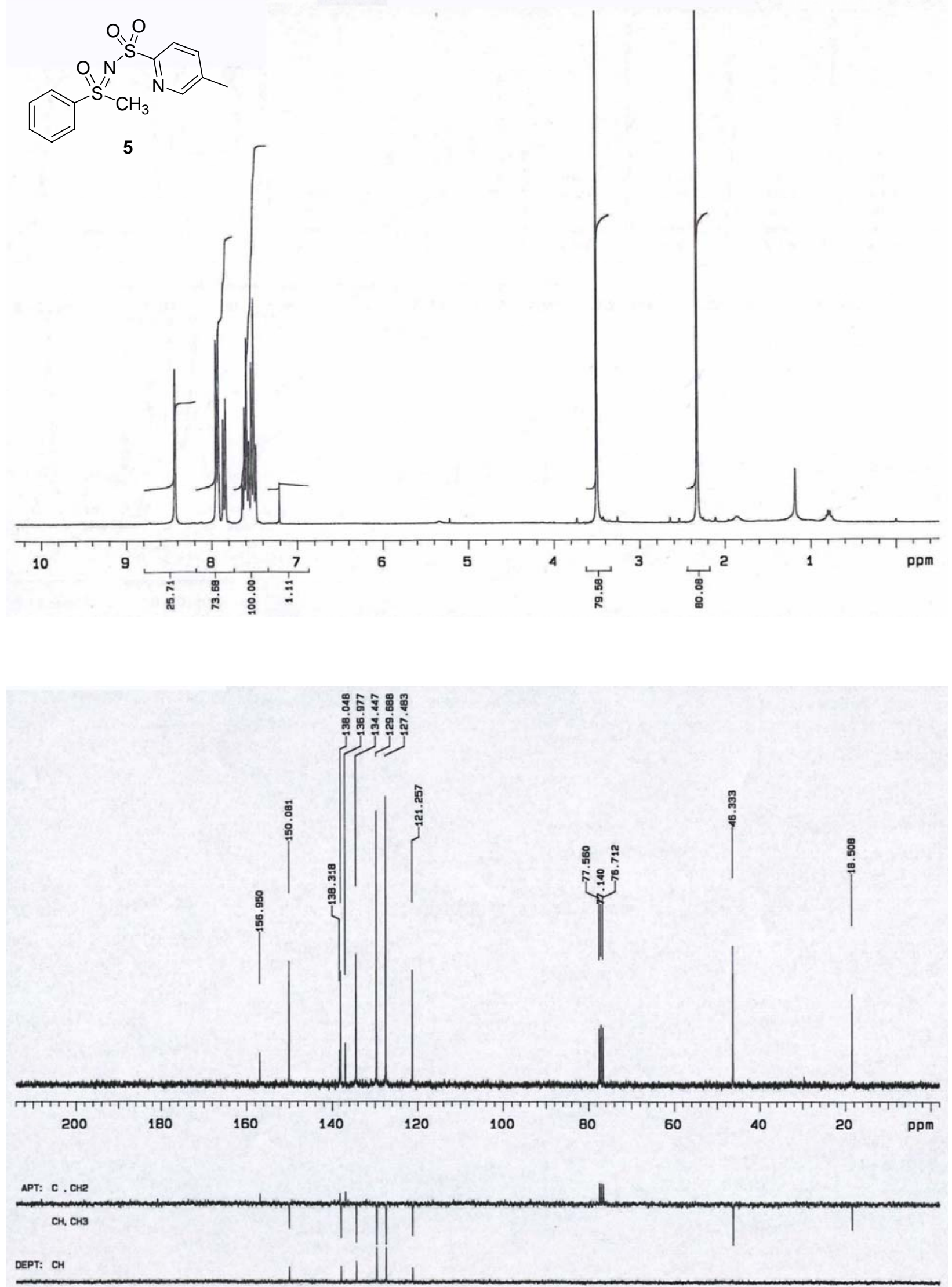

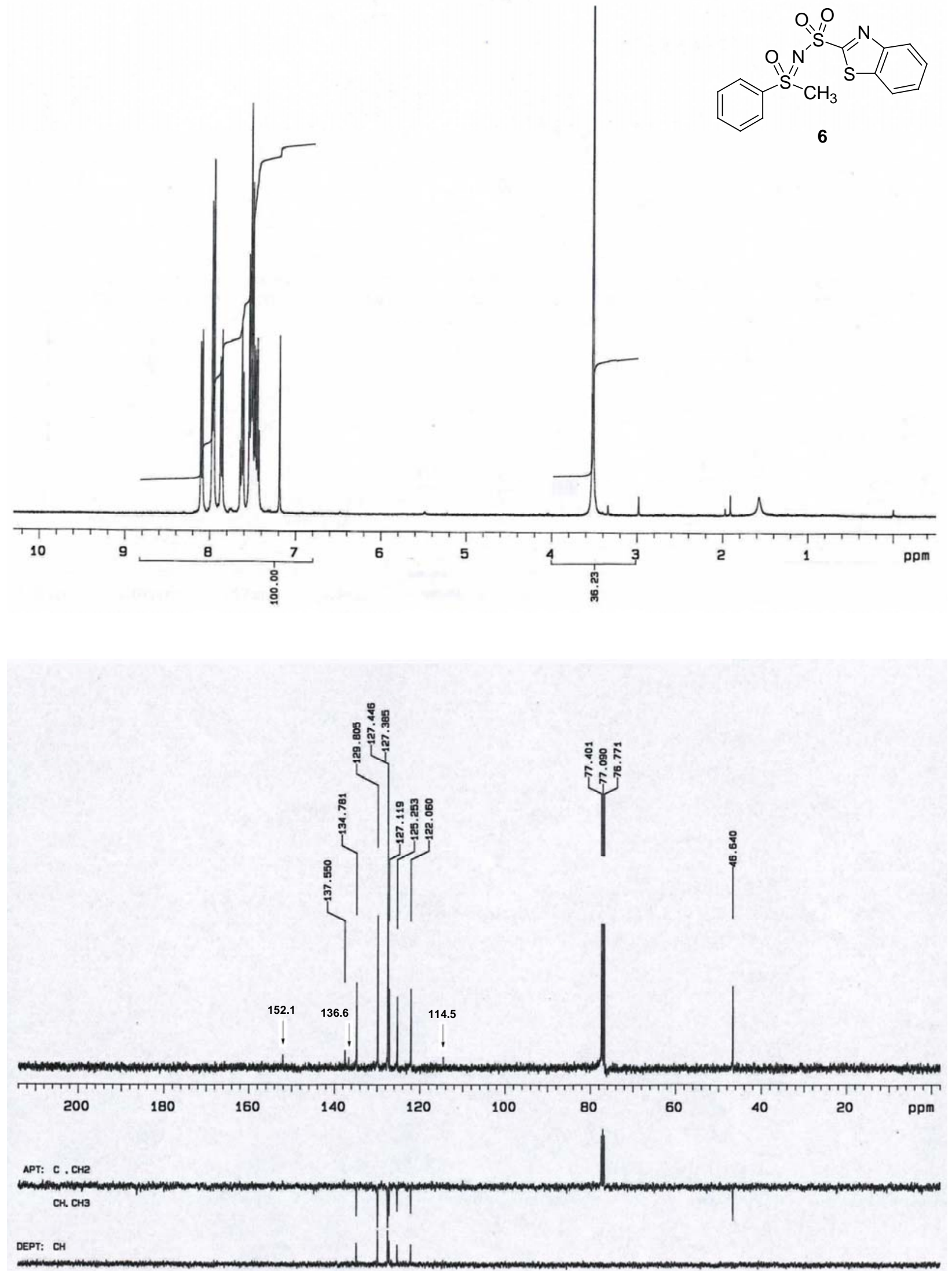


$$
\overbrace{}^{\mathrm{O} / \mathrm{N}-\mathrm{Ns}}
$$

7
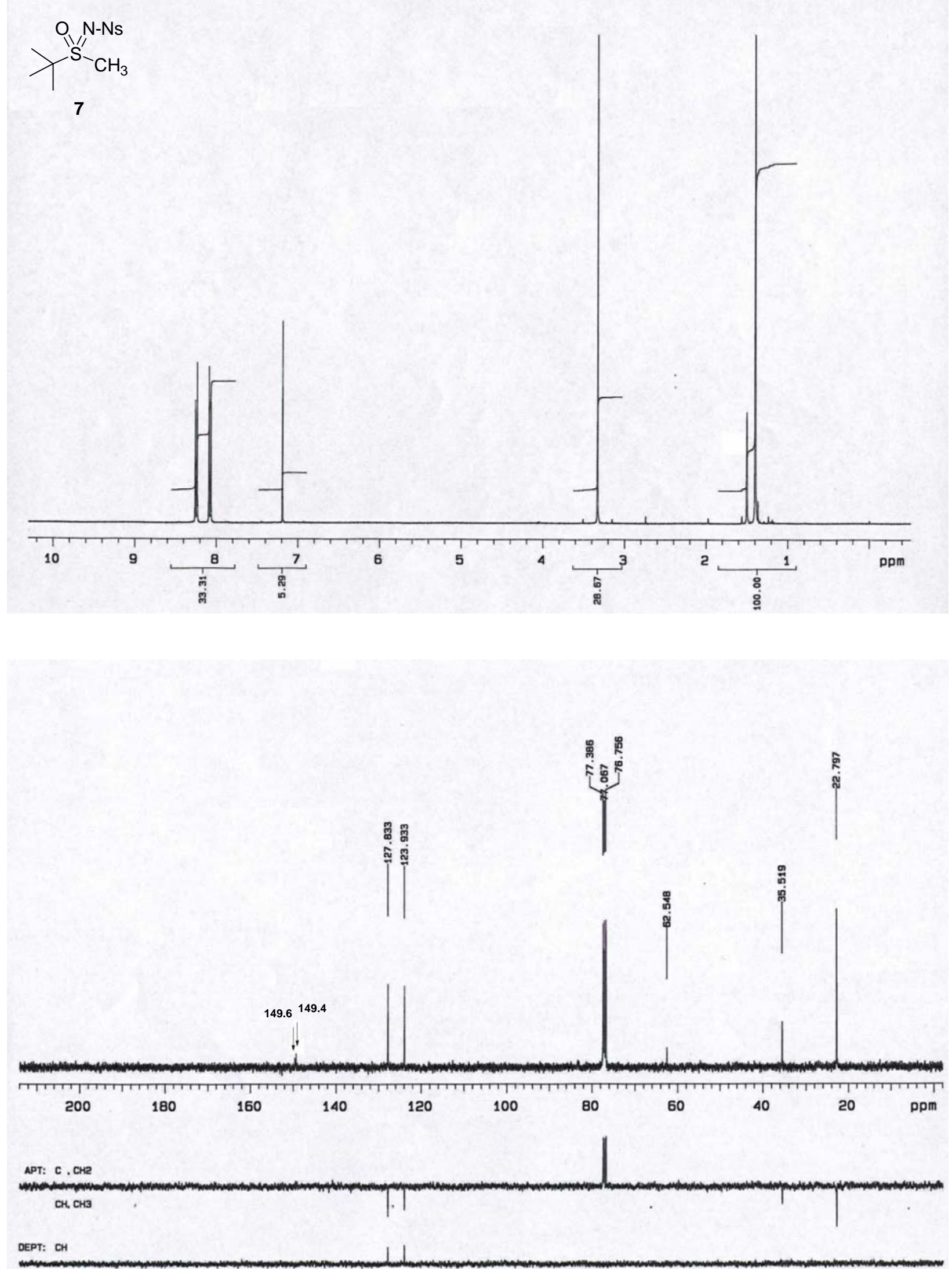

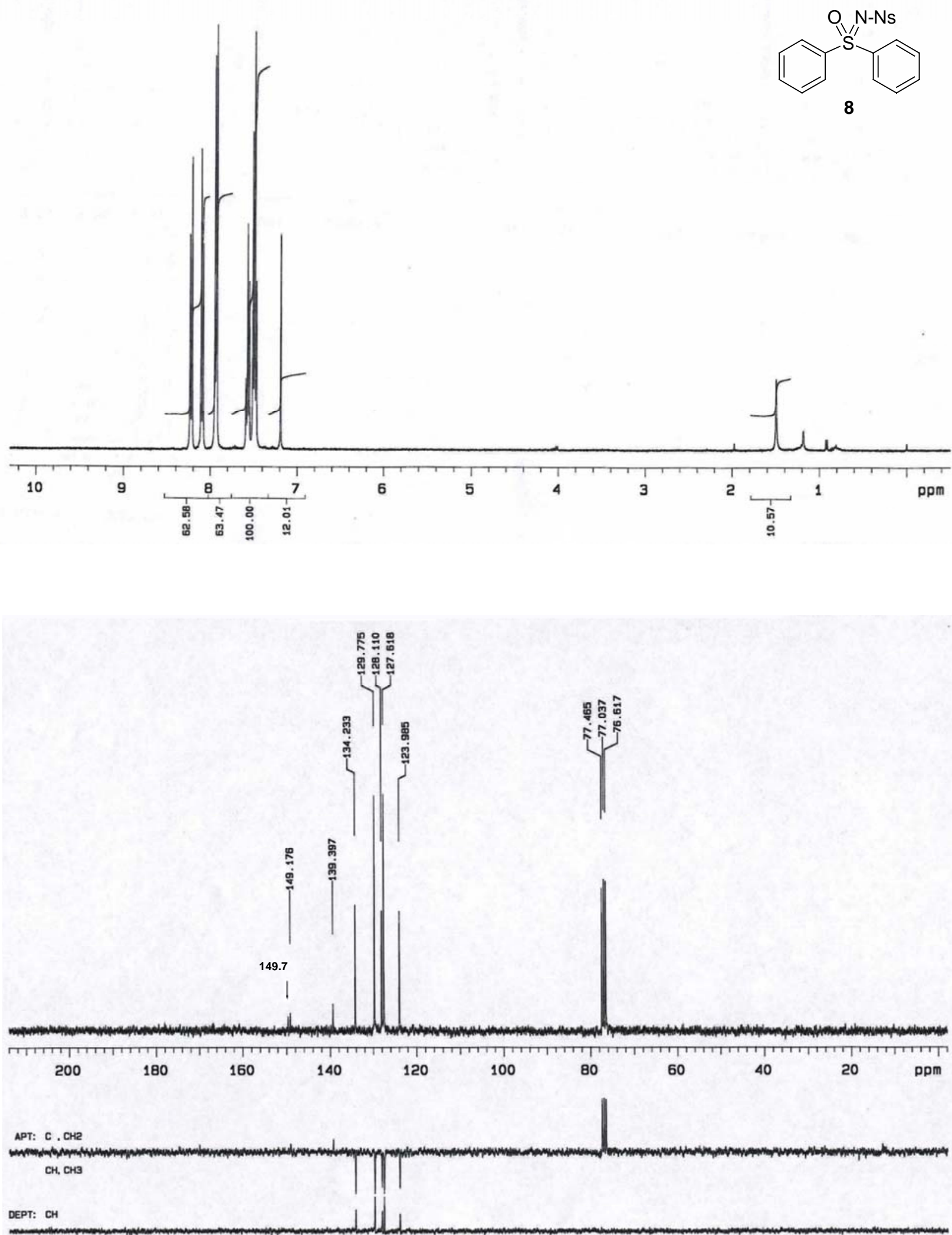

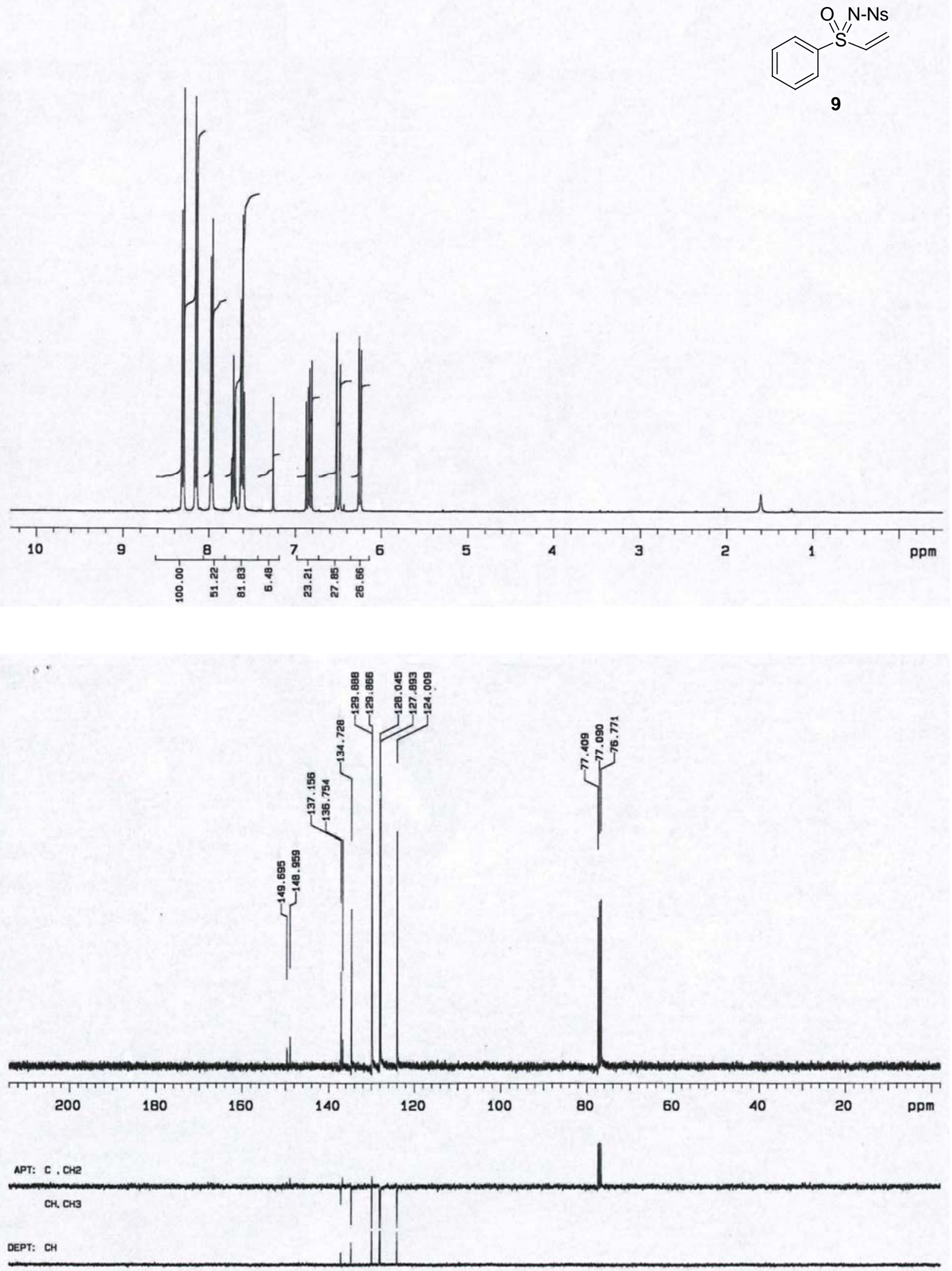
<smiles>[N+][SH]=S1(=O)CCCC1</smiles>

10

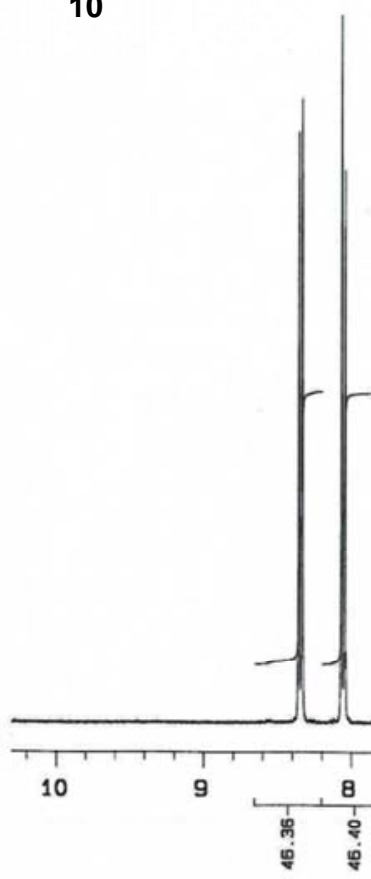

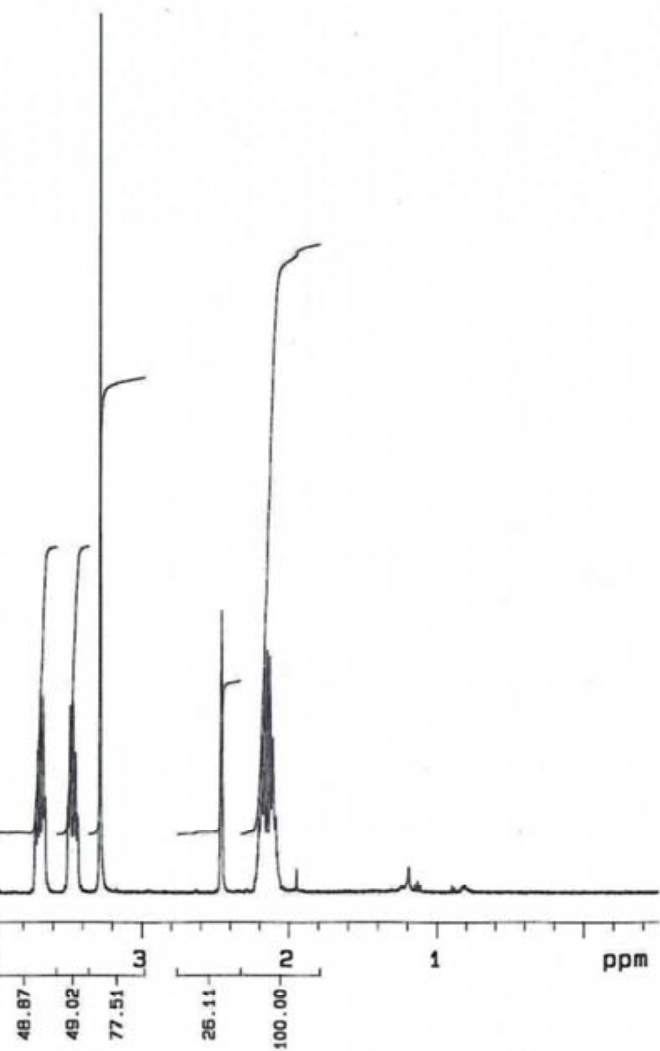

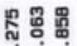
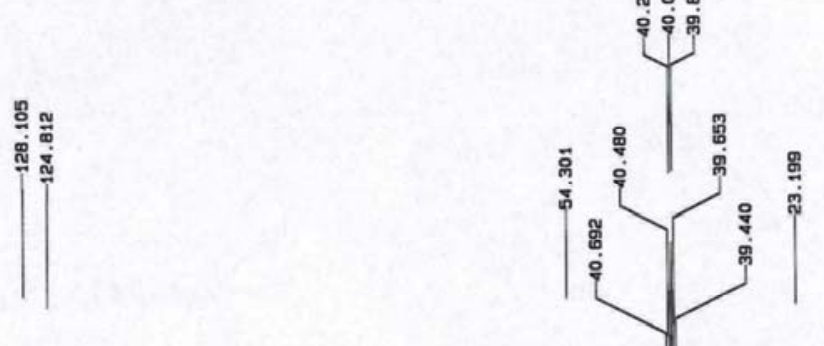

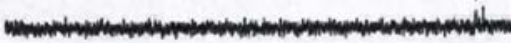

200

180

160

140

120

100

80

60

40

20

APT: C. CH2 $\mathrm{CH}, \mathrm{CH} 3$

DEPT: CH 


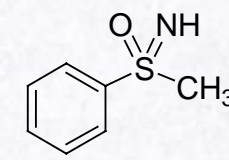

11
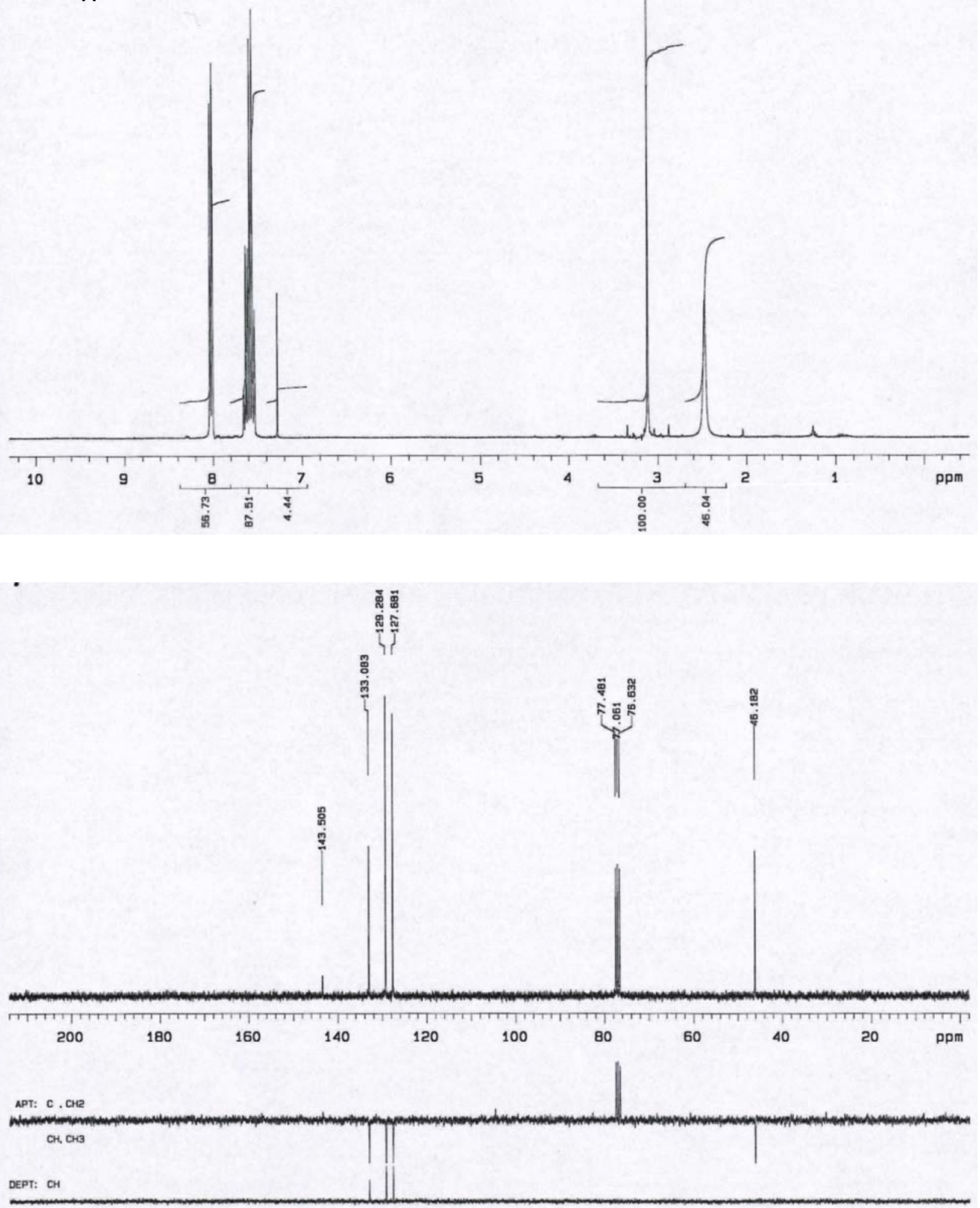

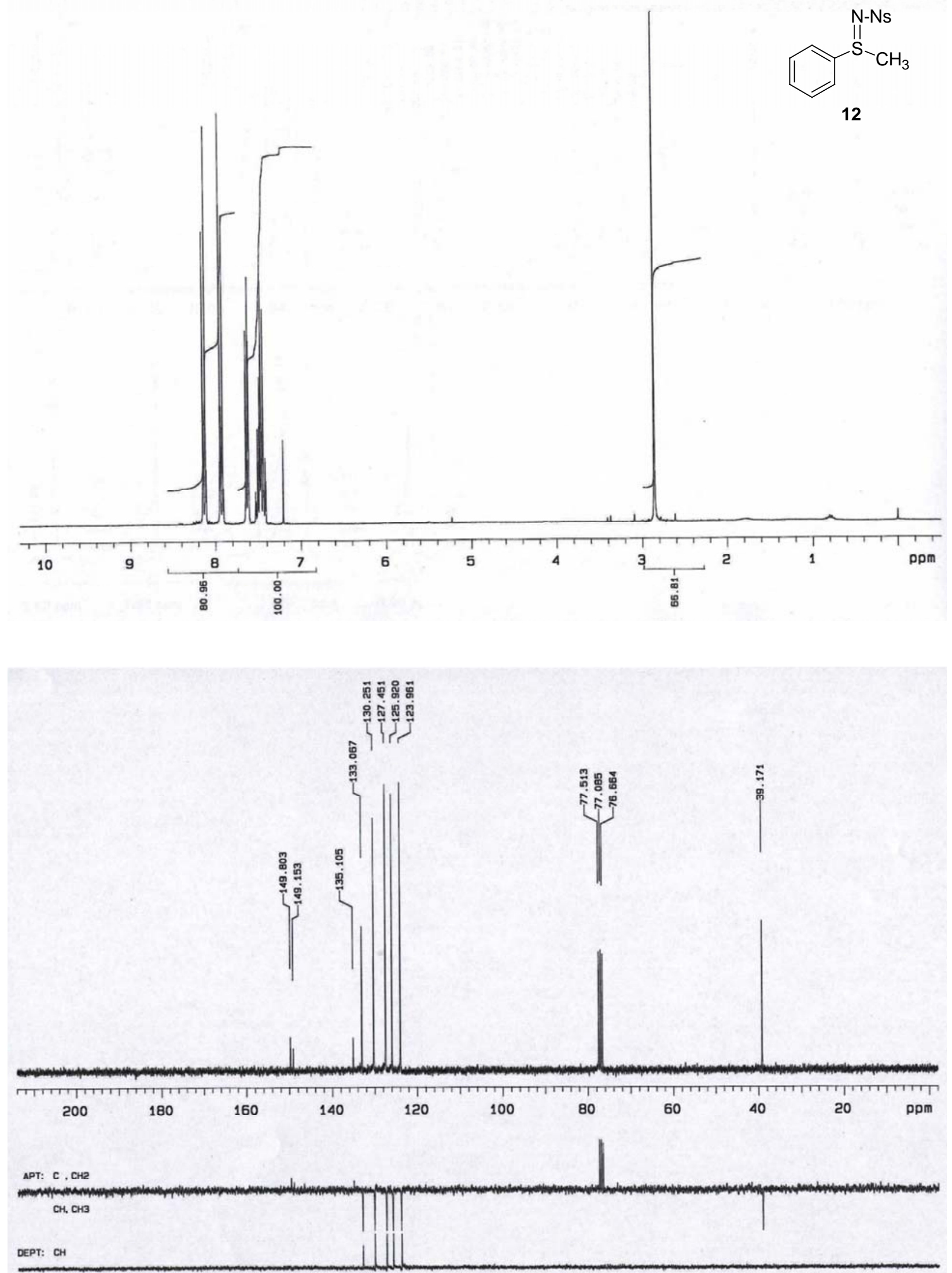
<smiles></smiles>

13
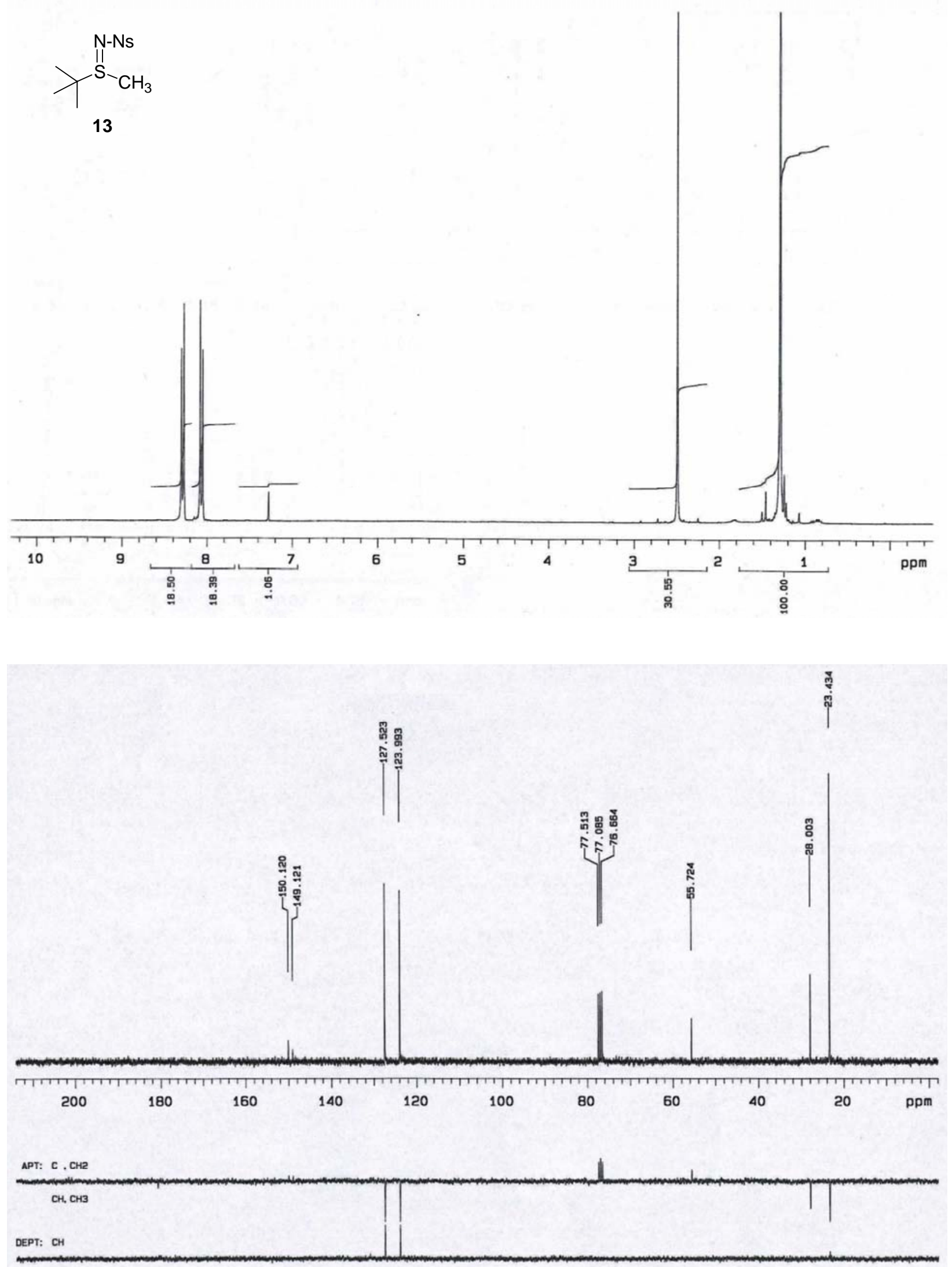

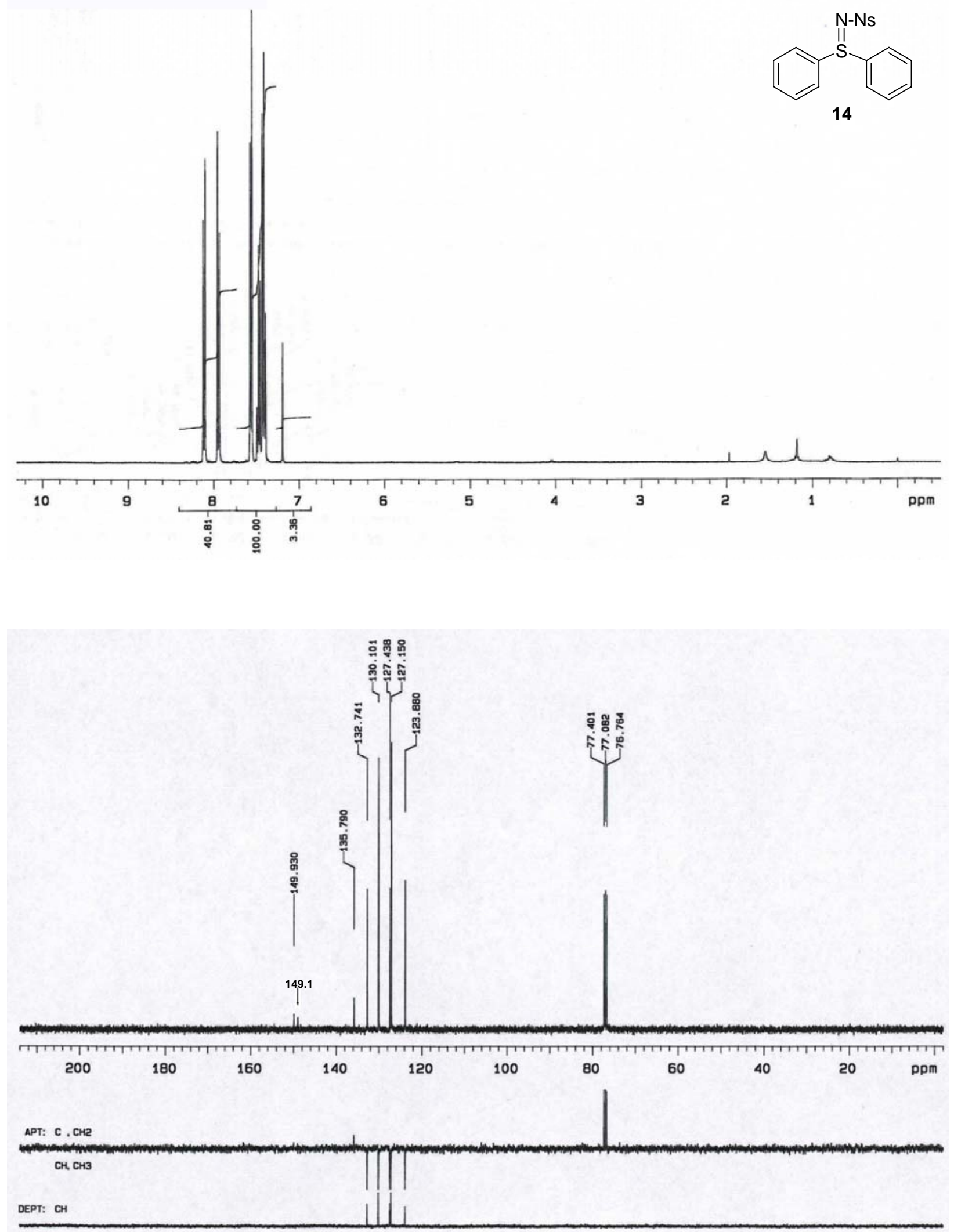

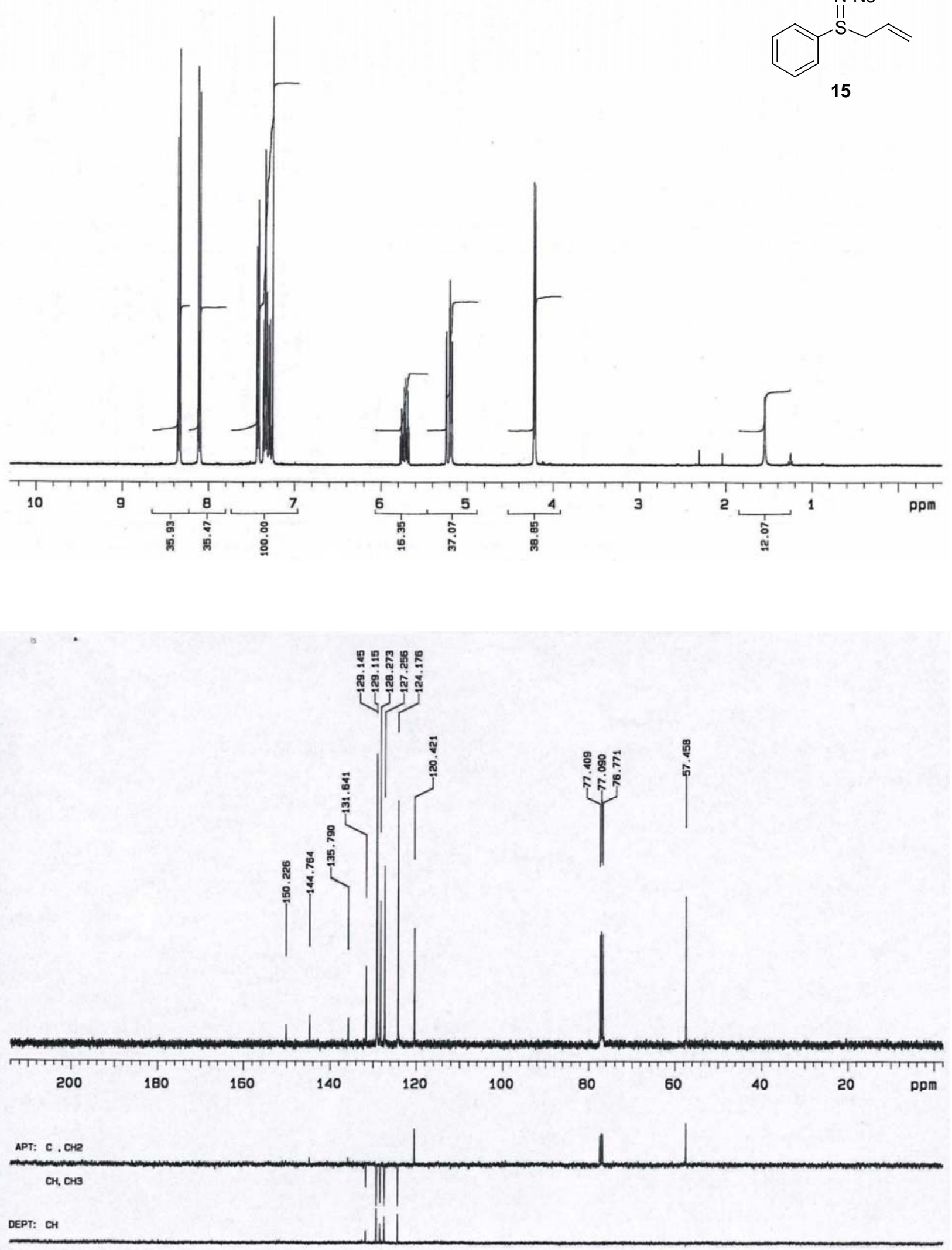
<smiles>Cc1cc(C)c(S(C)=NS(C)(=O)=O)c(C)c1</smiles>

16

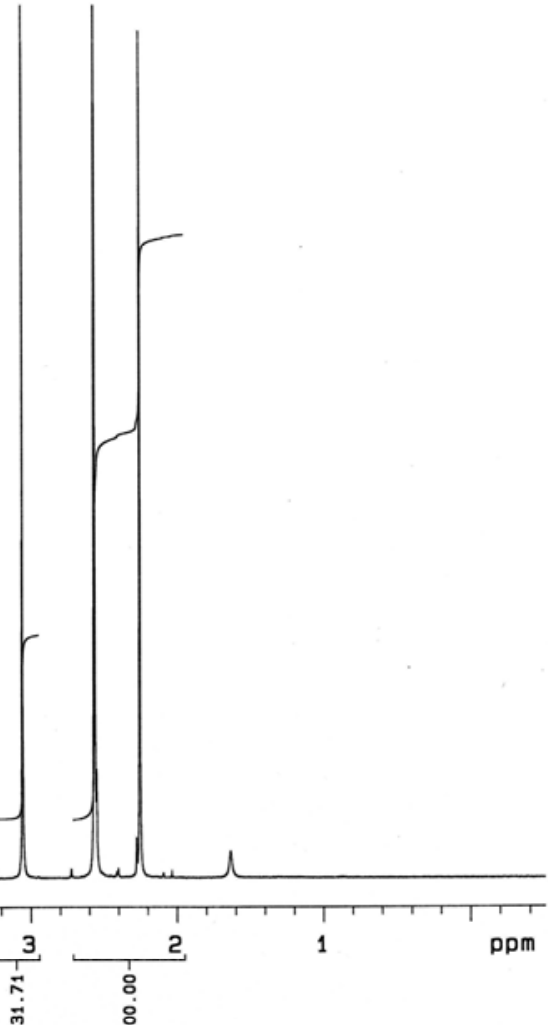

จุด

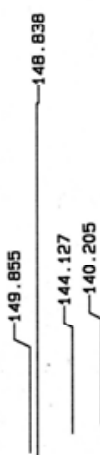

羿

ขึ

|
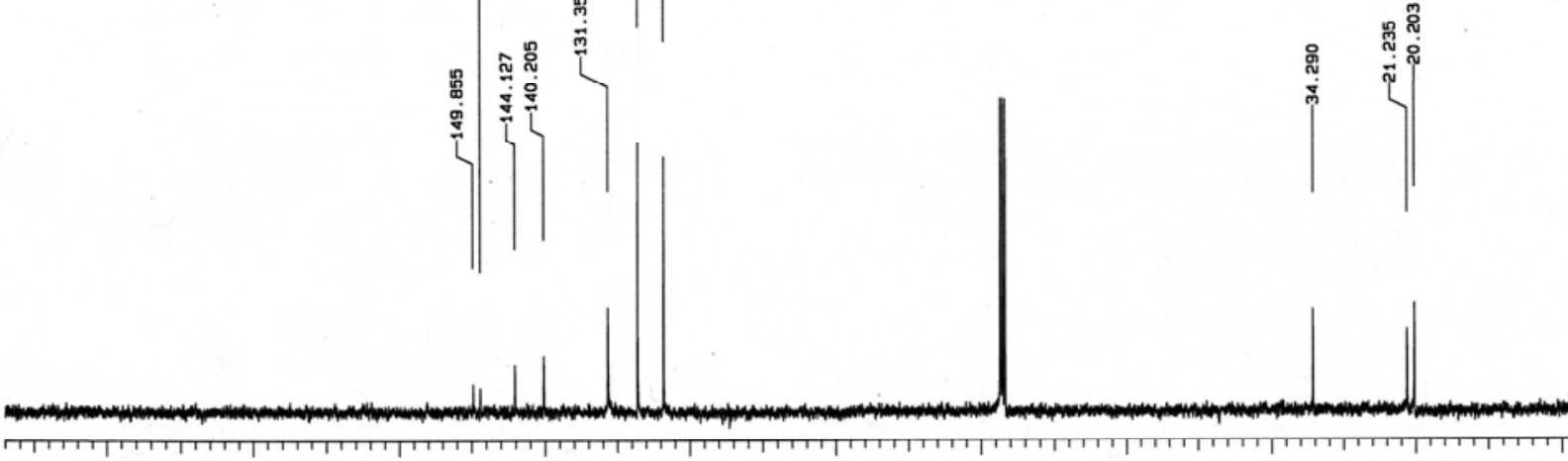
200 180 160 140 120 100 80 60 40 ppm APT: C , CH2 APT. C . CHe $\mathrm{CH}, \mathrm{CH} 3$

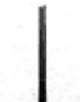

$\mathrm{TH}$

DEPT: CH 
<smiles>CS(=NS(=O)(=O)C(C)(C)C)c1ccccc1</smiles>

17

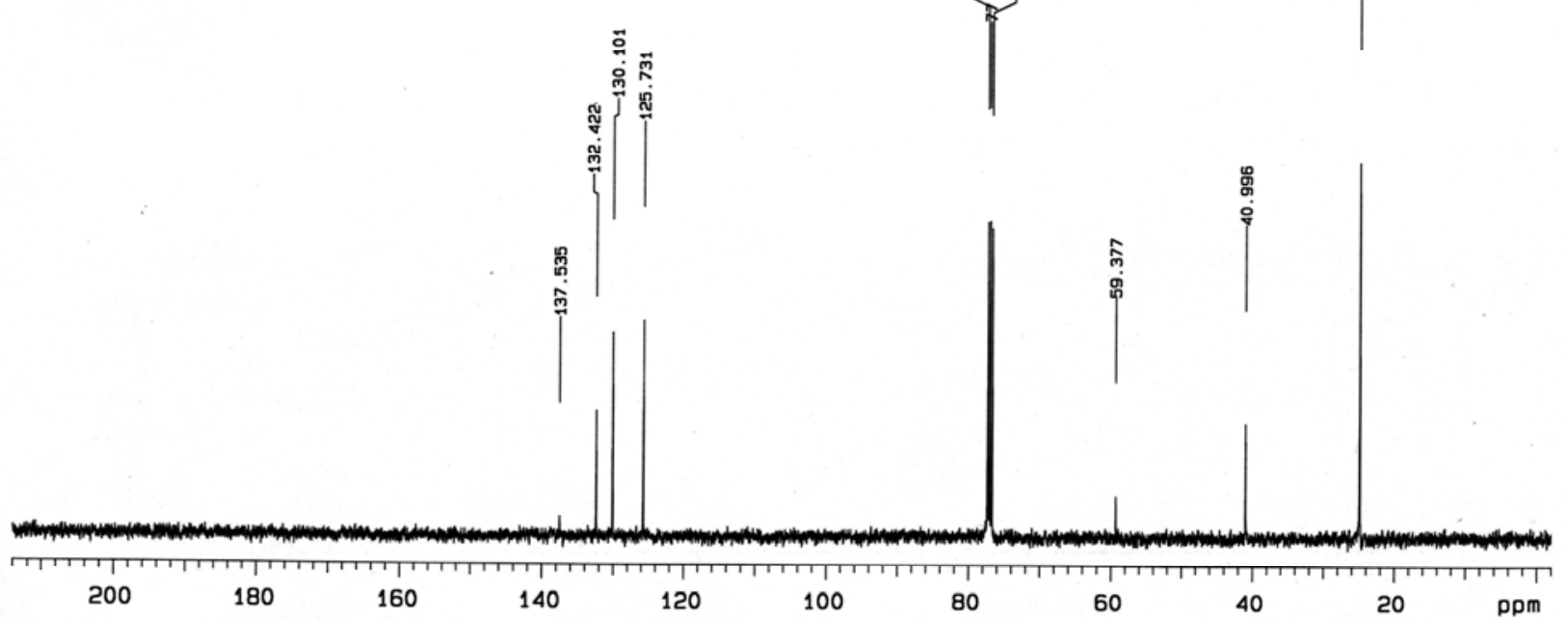

APT: C . CH2

APT: C.

DEPT: $\mathrm{CH}$

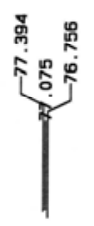

畐 